\title{
Analyzing quantum jumps of one and two atoms strongly coupled to an optical cavity
}

\author{
Sebastian Reick, ${ }^{1, *}$ Klaus Mølmer, ${ }^{2}$ Wolfgang Alt,${ }^{1}$ Martin Eckstein, ${ }^{1}$ Tobias Kampschulte, ${ }^{1}$ \\ Lingbo Kong, ${ }^{1, \dagger}$ René Reimann, ${ }^{1}$ Alexander Thobe, ${ }^{1, \ddagger}$ Artur Widera, ${ }^{1}$ and Dieter Meschede ${ }^{1}$ \\ 1 Institut für Angewandte Physik der Universität Bonn, Wegelerstrasse 8, 53115 Bonn, Germany \\ 2 Lundbeck Foundation Theoretical Center for Quantum System Research, \\ Department of Physics and Astronomy, University of Aarhus, DK-8000 Arhus C, Denmark \\ $\dagger$ Present address: Department of Physics, East Carolina University, Greenville, NC, USA \\ ${ }^{\ddagger}$ Present address: Institut für Laser-Physik der Universität Hamburg, \\ Luruper Chaussee 149, 22761 Hamburg, Germany and \\ *Corresponding author: sreick@uni-bonn.de
}

\begin{abstract}
We induce quantum jumps between the hyperfine ground states of one and two Cesium atoms, strongly coupled to the mode of a high-finesse optical resonator, and analyze the resulting random telegraph signals. We identify experimental parameters to deduce the atomic spin state nondestructively from the stream of photons transmitted through the cavity, achieving a compromise between a good signal-to-noise ratio and minimal measurement-induced perturbations. In order to extract optimum information about the spin dynamics from the photon count signal, a Bayesian update formalism is employed, which yields time-dependent probabilities for the atoms to be in either hyperfine state. We discuss the effect of super-Poissonian photon number distributions caused by atomic motion.
\end{abstract}

\section{INTRODUCTION}

Systems comprised of neutral atoms coupled to a single mode of a high-finesse resonator belong to the key modelsystems in quantum optics [1]. In the so called strong coupling limit an atom periodically exchanges its excitation energy with the resonator light field. In this case the dynamic evolution is governed by a priori entangled lightmatter quantum states, namely the combined dressed states of the atom-cavity system. Due to the symmetric interaction, described by the Janyes-Cummings Hamiltonian 2], information about the state of the system can be obtained from two complementary partial measurements: In the optical domain, experiments rely on the detection of photons emitted from the cavity [3 9 ], whereas in the microwave regime the quantum state of atoms transiting the cavity field is detected [10].

Optical cavity-QED systems are attractive for applications in quantum information science, e.g. for quantum networks. The successful demonstration of, for instance, the mapping of the coherent state of a traveling qubit (a photon) to the atomic state memory qubit [11] as well as single photon generation [12] are recent examples of significant progress in controlling the interaction of a single atom with the cavity field. For the creation of two-particle entangled states, promising proposals rely on either applying deterministic protocols [13 or measurement induced (probabilistic) projection [14, 15].

Not only for the application in quantum information science, but also more generally for the investigation of strongly interacting atom-cavity systems it is vital to understand the spin dynamics of one and especially more than one atom simultaneously coupled to the resonator field. In the work presented here we concentrate on the case of one and two atoms and investigate how maximum information about their hyperfine ground state can be retrieved from the stream of photons arriving at the detector. We outline and detail the identification of optimal experimental settings such as atom-cavity detuning.

Random telegraph signals, obtained by continuously observing quantum jumps between the spin states of a single atom, are analyzed by applying a Bayesian update formalism. In complementary experiments [16, 17], the photon number state (Fock state) of a microwave cavity field is interrogated by a stream of circular Rydbergatoms acting as quantum probes. There Bayesian analysis has proven to be a useful method of analysis, too. Random telegraph fluctuations are a universal phenomenon observed in many different fields, including a large variety of solid-state systems [18].

In order to study two-atom dynamics, we identified experimental parameters for which the intra-cavity intensity depends on the number of atoms in a specific spin state. The virtue of the Bayesian method is evident in analyzing the corresponding telegraph signals, for which the atomic state cannot be unambiguously deduced from the measured transmission signal because of technical limitations on the signal-to-noise ratio.

In our measurements we observe fluctuations in the transmission exceeding shot noise, which we attribute to thermal motion of the atom. We discuss the impact of this external dynamics on the performance of the Bayesian analysis.

\section{EXPERIMENTAL TECHNIQUES}

\section{A. Setup to trap and transport single atoms}

At the beginning of every experimental sequence, a controlled number of cesium (Cs) atoms is transferred from a magneto-optical trap (MOT) into a standing wave far-off-resonant dipole trap (FORT) with $\lambda_{\mathrm{FORT}}=1030$ $\mathrm{nm}$ and a trap depth of $U_{\mathrm{FORT}} \approx k_{B} \times 1 \mathrm{mK}$. This 


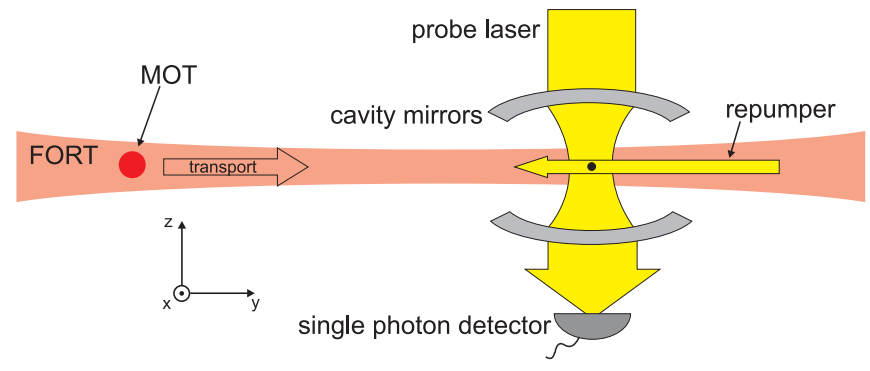

FIG. 1: Schematic setup of MOT, far-off resonance dipole trap (FORT) and cavity mirrors (not to scale). Details on the experimental setup and the stabilization of the cavity resonance frequency are given in [8].

trap acts as an "optical conveyor belt" [19] to transport atoms into the optical resonator. The fundamental $\mathrm{TEM}_{00}$ mode of the cavity has a diameter of $2 w_{0}=46$ $\mu \mathrm{m}$ and a length given by the mirror distance of $158 \mu \mathrm{m}$, the finesse is $\mathcal{F}=1.2 \times 10^{6}$.

A conceptual drawing of the main components is depicted in figure 1, for details on the cavity-setup and the stabilization scheme see [8]. To study the atom-cavity system, the transmission of a weak probe laser through the cavity is detected with a single-photon counting module (SPCM). Using a custom-build time-to-digital converter, we record - for each photon click - the time since the last click, where for our typical count-rates dead time effects are negligible. This list of click-intervals is then converted into a binned transmission signal by counting the detector clicks in each bin time interval $\Delta t_{b}$.

The total detection efficiency for the probe laser light, including absorption and scattering by the mirror coatings, losses at various optical elements along the optical path, and the quantum efficiency of the detector, amounts to $\eta=4.4 \%$, which is a threefold improvement compared to our earlier work presented in [9]. The main challenge was to optimize the separation of probe- and stabilization-laser, with typical powers of a few $10^{-15}$ and $10^{-6}$ Watts, respectively. In a first step, they are separated by their carefully adjusted orthogonal polarizations. Improved spectral filtering was achieved by replacing a standard ruled diffraction grating with a volume holographic grating, allowing us to omit an additional interference filter used before, while still achieving a total suppression of the stabilization laser to better than $10^{-8}$.

The probe laser frequency is set close to the $|F=4\rangle \rightarrow\left|F^{\prime}=5\right\rangle$ transition of the Cs $D_{2}$ line, where $F$ is the total angular momentum quantum number. For this transition, the important parameters of the atomcavity system are $(g, \kappa, \gamma)=2 \pi \times(13.1,0.4,2.6) \mathrm{MHz}$, where $g$ is the nominal coupling strength for an atom at the position of maximum coupling, $\kappa$ is the cavity field decay rate, and $\gamma$ is the atomic dipole decay rate. Since in our setup the birefringent splitting of the cavity resonances is larger than the cavity linewidth, the cavity field is always linearly polarized, causing a distribution (a)

(b)

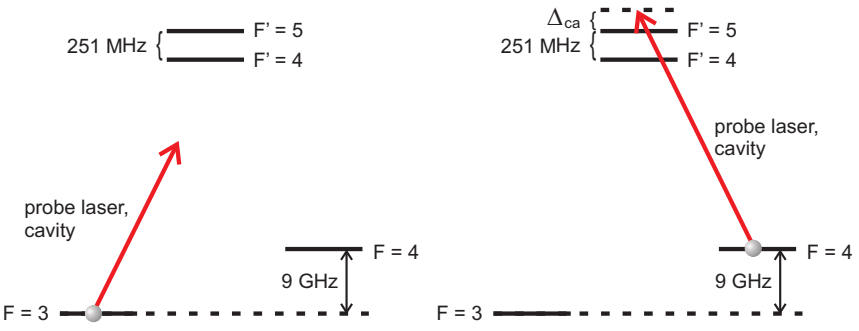

FIG. 2: Simplified Cs level scheme. (a) An atom in $|F=3\rangle$ is so far detuned from the cavity resonance that it does not alter its transmission. (b) If the atom is in $|F=4\rangle$, it changes the transmission, depending on the cavity-atom detuning $\Delta_{c a}$ and the coupling strength $g$.

of the atomic population over all Zeeman sublevels due to photon scattering by the probe laser. Thus the coupling strength $g$ given above is obtained from a weighted average over all couplings $g\left(m_{F}\right)$, based on the steady state $m_{F}$ distribution for linearly polarized optical pumping 20, 21]. With the single-atom cooperativity parameter $C_{1}=g^{2} /(2 \kappa \gamma) \gg 1$, our system is in the strong coupling regime, where already a single atom significantly influences the cavity spectrum.

\section{B. Nondestructive state detection}

In our system the two long-lived hyperfine groundstates $|F=3\rangle$ and $|F=4\rangle$ serve as qubit states [22]. For the coupled atom-cavity system we measure this state by tuning the cavity close to the $|F=4\rangle \rightarrow\left|F^{\prime}=5\right\rangle$ transition, where only an atom in the $|F=4\rangle$ state leads to a drop in the transmission, while an atom in $|F=3\rangle$ is so far detuned (around $9.2 \mathrm{GHz}$ ) that it effectively decouples from the system and does not influence the cavity transmission, see fig. 2

The probe laser with angular frequency $\omega_{p}$ is initially tuned to the resonance frequency of the empty cavity $\omega_{c}=\omega_{p}$, so when an atom in $|F=4\rangle$ is inserted into the cavity the transmission is reduced to a level which depends on the detuning $\Delta_{c a}=\omega_{c}-\omega_{a}$, where $\omega_{a}$ is the angular frequency of the atomic $|F=4\rangle \rightarrow\left|F^{\prime}=5\right\rangle$ transition, including the AC-Stark shift induced by the FORT potential. To experimentally distinguish between an atom in $|F=3\rangle$ and an atom lost from the trap, which both result in the same transmission signal, a repumping laser resonant with the $|F=3\rangle \rightarrow\left|F^{\prime}=4\right\rangle$ transition can be applied from the side which brings the atom in $|F=3\rangle$ back to the $|F=4\rangle \rightarrow\left|F^{\prime}=5\right\rangle$ cycle. Thus for an empty cavity the transmission would remain unchanged, while for an atom still present in the cavity the transmission would drop again. 


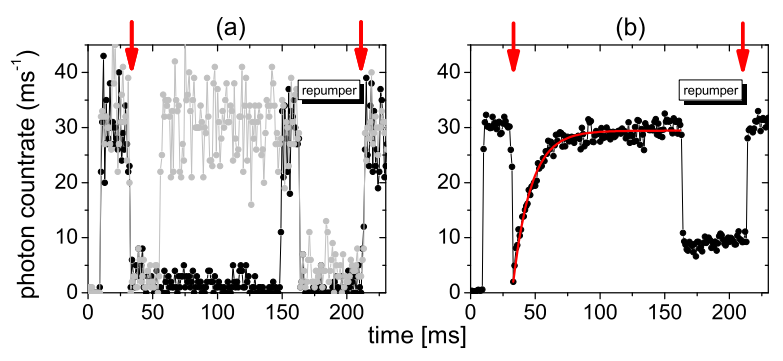

FIG. 3: (a): Black and grey curves show two single traces of quantum jump measurements. The arrows indicate insertion and removal of an atom. At the end of the sequence, the repumper is switched on again to check that the atom was not lost. (b): Ensemble average over 31 single traces. The average dwell time $R_{43}^{-1}$ is obtained from the exponential fit. The averaged transmission level at the end of the sequence, when the repumper is switched on, is higher than the initial drop, indicating a lower average coupling strength. This could be caused by increased thermal motion, a re-distribution over different $m_{F}$-levels, or a combination of both effects.

\section{SINGLE ATOM SPIN DYNAMICS}

If the state detection technique described above gave the same result for an unlimited series of state measurements, it would be a perfect projective quantum nondemolition (QND) measurement [23 25], assuming the system is otherwise unperturbed. However, in our situation the same laser that we use to detect the atomic state (the probe laser) can change it via inelastic hyperfine-statechanging Raman scattering. An atom in the $|F=4\rangle$ groundstate can thus be transferred to $|F=3\rangle$ via the $\left|F^{\prime}=3\right\rangle$ and $\left|F^{\prime}=4\right\rangle$ excited states, thereby undergoing a quantum jump [26 30].

\section{A. Quantum jump rate and transmission level as a function of detuning}

To experimentally determine the rate $R_{43}$ of probelaser-induced transitions from $|F=4\rangle$ to $|F=3\rangle$ and to identify optimum experimental conditions, we performed the following measurement: An atom, optically pumped into $|F=4\rangle$, is transported into the cavity center, causing a drop of the cavity transmission, see fig. 3 (a). Since no repumper is applied, probe-laser scattering causes a spontaneous transition to $|F=3\rangle$ after some time, visible as an instantaneous rise in transmission back to the empty cavity level. To check whether the rise in transmission is really due to a quantum jump and not caused by atom loss, the repumper is switched on at the end of the sequence as discussed above.

For each experimental realization, the quantum jump occurs at a random point in time, see fig. 3 (a) for two example traces. Since the rate of state transitions is time independent, the ensemble average plotted in fig. 3 (b) reveals the expected exponential curve with the time con-
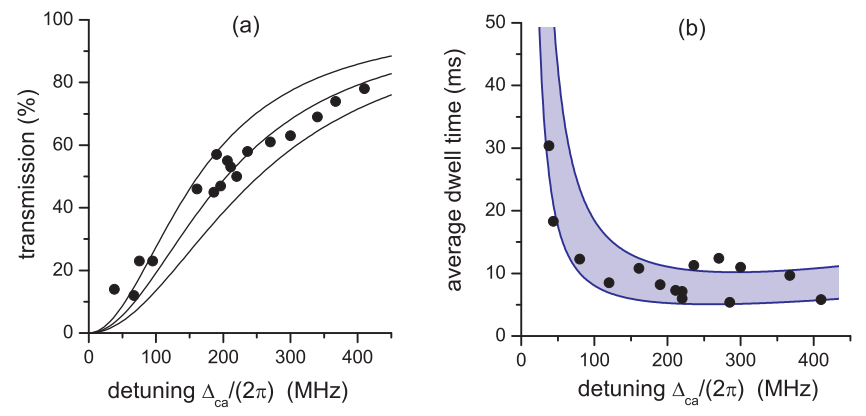

FIG. 4: (a) Normalized one-atom-transmission as a function of the cavity-atom detuning $\Delta_{c a}$. The solid lines are calculated for an atom at rest with an effective coupling strength of $g_{\text {eff }} /(2 \pi)=8,9$, and $10 \mathrm{MHz}$ for the upper, middle, and lower curve, respectively. (b) Average dwell time $R_{43}^{-1}$ as a function of detuning. The shaded area is the result of a theoretical model taking motion of the atom into account, and the range of values represents our limited knowledge about the exact distribution over the Zeeman-sublevels.

stant being the average dwell time $R_{43}^{-1}$.

This average dwell time was measured for a wide range of detunings $\Delta_{c a} /(2 \pi)=38 \ldots 410 \mathrm{MHz}$. For the same settings, but with the repumper constantly applied, we measured the transmission level $T_{1}$, defined as the photon count rate with one atom in $|F=4\rangle$ coupled to the cavity, normalized to the empty cavity signal. The results of

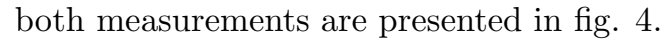

In order to describe our measurements with a simplified analytical model, we consider a two-level atom at rest with the probe-laser being resonant with the empty cavity $\left(\omega_{p}=\omega_{c}\right)$. In the weak excitation regime, the oneatom-transmission level can be expressed analytically as [31]

$$
T_{1}\left(\Delta_{c a}, g_{\mathrm{eff}}\right)=\frac{\kappa^{2}\left(\Delta_{c a}^{2}+\gamma^{2}\right)}{\left(\gamma \kappa+g_{\mathrm{eff}}^{2}\right)^{2}+\left(\Delta_{c a} \kappa\right)^{2}} .
$$

The distribution over Zeeman sublevels, thermal motion of the atom, AC-Stark shift variations, and other conceivable perturbations are all accounted for by an effective coupling strength $g_{\text {eff. }}$. It is defined by Eq. (1D) in such a way that a stationary two-level atom with a coupling strength of $g_{\text {eff }}$ would yield the experimentally measured transmission level. The solid lines in fig. 4 (a) are calculated according to Eq. (11) with $g_{\text {eff }} /(2 \pi)=8,9$, and $10 \mathrm{MHz}$, and this range of effective couplings describes the data reasonably well. We attribute the difference between the nominal coupling strength of $g /(2 \pi)=13.1$ $\mathrm{MHz}$ and $g_{\text {eff }}$ mainly to thermal motion of the atom.

To describe the measured average dwell times theoretically, see fig. 4 (b), $R_{43}$ is calculated as a function of detuning using the Kramers-Heisenberg-formula [32]. For this calculation, one has to treat the distribution over all Zeeman sublevels and thermal motion separately, since this situation cannot be modeled as a two-level system with an effective coupling. The measured data agree satisfactorily with the theoretical model, confirming that the 

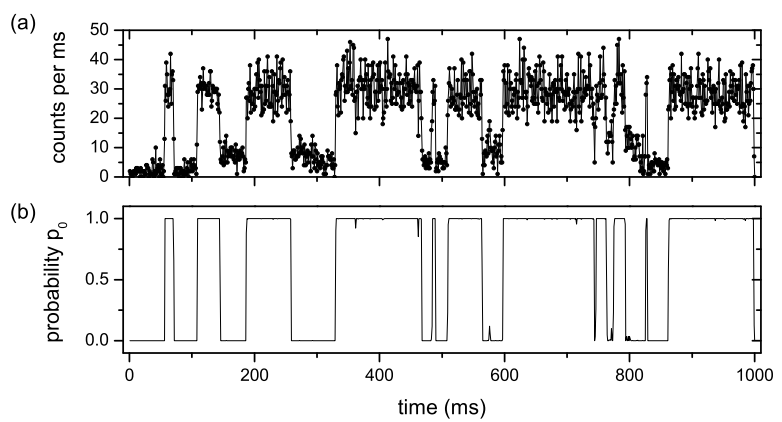

FIG. 5: (a) Random telegraph signal for one atom coupled to the cavity. (b) Bayes analysis yielding $p_{0}(t)$, i.e. the probability to be in $|F=3\rangle$.

best approximation to a projective QND-measurement with longest dwell times is close to resonance. A practical limitation is that stable coupling was never observed for detunings $\Delta_{c a} \lesssim 2 \pi \times 30 \mathrm{MHz}$, probably due to cavity cooling becoming less effective [33, 34].

\section{B. Statistical analysis of single-atom random telegraph signals}

In the experiments discussed so far, the repumping laser was either switched off or its intensity was adjusted such that an atom off-resonantly transferred to $|F=3\rangle$ was pumped back to $|F=4\rangle$ immediately, compared to all relevant time scales in our experiment. In contrast, for the measurements presented in the following, we deliberately attenuated the continuously applied repumping laser to a level at which the transfer rate $R_{34}$ from $|F=3\rangle$ to $|F=4\rangle$ was comparable to $R_{43}$. Therefore, the resulting quantum jumps occur in both directions on a similar timescale of several milliseconds and are thus detectable as a random telegraph signal, see fig. 5] (a) for an example trace.

We quantify our knowledge about the atom's hyperfine spin by probabilities assigned to the different atomic states. For the following discussion we introduce the parameter $\alpha$ to denote the number of atoms in $|F=4\rangle$. In this section $\alpha$ attains only the values 0 and 1 , whereas the case of two atoms (see section [V]) also permits the value $\alpha=2$. Internal state changes of a single atom are transitions between the two states $\alpha=0,1$, and they occur with the rates $R_{10}$ and $R_{01}$, which are identical to $R_{43}$ and $R_{34}$, respectively. Although we imagine the transitions to occur randomly and at discrete instances of time, the probabilities for the atom to occupy the different states change in a continuous manner governed by the following rate equations:

$$
\begin{aligned}
& \frac{\mathrm{d} p_{0}(t)}{\mathrm{d} t}=-R_{01} p_{0}(t)+R_{10} p_{1}(t) \\
& \frac{\mathrm{d} p_{1}(t)}{\mathrm{d} t}=-R_{10} p_{1}(t)+R_{01} p_{0}(t)=-\frac{\mathrm{d} p_{0}(t)}{\mathrm{d} t} .
\end{aligned}
$$

The average steady-state probabilities $\bar{p}_{0}$ and $\bar{p}_{1}$ are obtained by setting $\frac{\mathrm{d} p_{0}(t)}{\mathrm{d} t}=\frac{\mathrm{d} p_{1}(t)}{\mathrm{d} t}=0$ and using $\bar{p}_{0}+\bar{p}_{1}=$ 1. The solutions are thus given by the ratio between the transition rates:

$$
\begin{aligned}
\bar{p}_{0} & =\frac{R_{10}}{R_{10}+R_{01}}, \\
\bar{p}_{1} & =\frac{R_{01}}{R_{10}+R_{01}} .
\end{aligned}
$$

The average probabilities and thus the ratio of the rates $R_{10}$ and $R_{01}$ can therefore be obtained from photon count histograms by the following procedure: Along with the telegraph signals, transmission traces for an empty cavity $(\alpha=0)$, and for one continuously coupled atom $(\alpha=1)$ were measured for otherwise identical settings. From these three sets of data, normalized photon count histograms $\mathcal{P}(n), \mathcal{P}(n \mid 0)$ and $\mathcal{P}(n \mid 1)$ are computed, with $n$ being the number of photons detected per binning time $\Delta t_{b}=1 \mathrm{~ms}$. Here and for the remaining discussion, $\mathcal{P}$ always refers to photon-count probabilities, while $p_{\alpha}(t)$ indicates spin-state probabilities.

Since the telegraph signal is expected to represent the atomic system jumping between the different states, the associated accumulated histogram of photon counts should be a weighted fit

$$
\mathcal{P}(n)=\bar{p}_{0} \mathcal{P}(n \mid 0)+\left(1-\bar{p}_{0}\right) \mathcal{P}(n \mid 1),
$$

of the independently measured histograms $\mathcal{P}(n \mid \alpha)$ for the two atomic states. Treating $\bar{p}_{0}$ as a fitting parameter yields $\bar{p}_{0}=0.64$ and $\bar{p}_{1}=0.36$.

In order to extract the transition rates, we note that the jumping of the atom between two different states with different transmission properties causes characteristic fluctuations in the number of detection events obtained in different time bins, $n(t)$ and $n(t+\tau)$, which become visible in the second-order correlation function $g^{(2)}(\tau)$. Assuming Poissonian count distributions, an analysis of the rate equations yields [28]

$g^{(2)}(\tau)=\frac{\langle n(t) n(t+\tau)\rangle}{\langle n(t)\rangle\langle n(t+\tau)\rangle} \propto \exp \left(-\left(R_{10}+R_{01}\right) \tau\right)$ for $\tau>0$.

The histogram of the telegraph signal and the $g^{(2)}$ function are plotted in figure 6] (a) and (b), respectively. From an exponential fit of the correlation function, we get $R_{10}+R_{01}=50 \mathrm{~s}^{-1}$, therefore we obtain $R_{10}=40 \mathrm{~s}^{-1}$ and $R_{01}=18 \mathrm{~s}^{-1}$ using $\bar{p}_{0}, \bar{p}_{1}$, and Eqs. (4) and (5).

In the discussion above, we assumed that the state of the atom can be described by the two states $\alpha=0$ and $\alpha=1$ alone, each leading to a Poissonian distribution $\mathcal{P}(n \mid \alpha)$ of the photon count rate. For $\alpha=0$ this is verified by the measurement: The right peak of the measured histogram in figure 6 (a) agrees with a Poissonian distribution of the the same average count rate. Thus the state detection for $\alpha=0$, with the transmission being equal to the empty-cavity case, is essentially shot-noise limited and residual frequency or intensity fluctuations of the probe-laser can be neglected. 

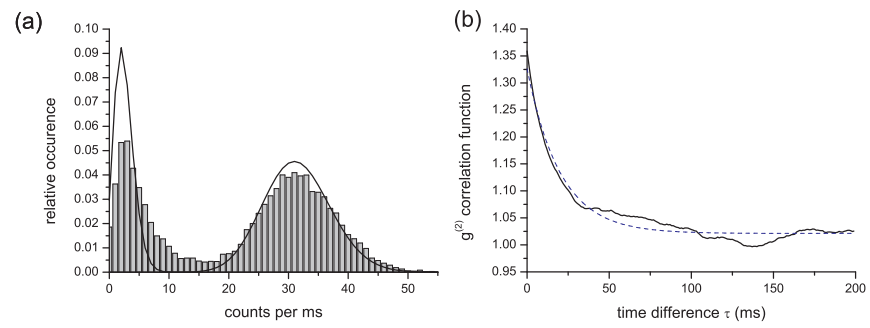

FIG. 6: (a) Normalized histogram extracted from 13 telegraph signals of $1000 \mathrm{~ms}$ duration each, binned with $\Delta t_{b}=1$ ms. The solid line is a Poissonian distribution with the same maximum count rate. (b) Averaged second order correlation function $g^{(2)}$ for the same set of telegraph signals. The blue dashed line is an exponential fit yielding the time constant $\left(R_{10}+R_{01}\right)^{-1}=20 \mathrm{~ms}$.

However, comparing the photon count histogram $\mathcal{P}(n \mid 1)$ with the Poissonian distribution (left peak of the histogram in fig. 6] (a)) indicates super-Poissonian fluctuations. We attribute these mainly to thermal motion of the atom: The coupling constant $g$ follows the cavity mode function, i.e. $g(\mathbf{r})=g_{0} \psi(\mathbf{r})$, which in turn leads to a transmission level $T_{1}(\mathbf{r})$, depending on the atomic position, according to Eq. (11).

In later sections of this paper we shall discuss candidates for a more complete theoretical analysis of this dynamics. At this point, we pursue a pragmatic approach and still extract the atomic transition rates from the correlation function as stated by Eq. (7), because this relation does not rely strongly on the Poissonian character of the signal. Furthermore, the exact values of the rates are not the main result of this work and do not convey fundamental physical insight, since they are determined by the intensities of the probe and repumping laser. They rather constitute parameters in the following statistical analysis.

To quantify the knowledge about the atomic state that we obtain from the measured telegraph signals, we use a Bayesian approach in analyzing the data. The philosophy behind this approach is that we assign probabilities to the possible states $\alpha=0,1$ of the atom, and acknowledge that these probabilities merely reflect our incomplete knowledge about the system, unless one of the probabilities is unity. Due to the atomic transitions which occur without our direct noticing, the probabilities of the unobserved system obey the rate equations (2) and (3), but since the cavity transmission depends on the atomic state, we learn about the atomic state from the observed photon count record.

The probabilities $p_{\alpha}$ are thus calculated step-wise from the incremental information obtained in every time bin of the measured telegraph signal. Let $n\left(t_{i}\right)$ be the number of photons detected during the interval $\left[t_{i}-\Delta t_{b} / 2, t_{i}+\right.$ $\left.\Delta t_{b} / 2\right]$, where the binning time $\Delta t_{b}$ is fixed to $1 \mathrm{~ms}$ for the following analysis. With $p_{\alpha}\left(t_{i}\right)$ we refer to the probability for an atom to be in the state $\alpha$ in the midpoint of the aforementioned interval. Assuming that the atomic state probabilities in the previous time bin $p_{\alpha}\left(t_{i-1}\right)$ are known, the probabilities $p_{\alpha}\left(t_{i}\right)$ are estimated by first evolving their values according to the rate equations (2) and (3). In a linear approximation for $R_{x} \Delta t_{b} \ll 1$, where $R_{x}=$ $\max \left(R_{10}, R_{01}\right)$, this leads to

$$
\begin{aligned}
& \tilde{p}_{0}\left(t_{i}\right)=p_{0}\left(t_{i-1}\right)+\left(R_{10} p_{1}\left(t_{i-1}\right)-R_{01} p_{0}\left(t_{i-1}\right)\right) \Delta t_{b}(8) \\
& \tilde{p}_{1}\left(t_{i}\right)=p_{1}\left(t_{i-1}\right)+\left(R_{01} p_{0}\left(t_{i-1}\right)-R_{10} p_{1}\left(t_{i-1}\right)\right) \Delta t_{b}(9)
\end{aligned}
$$

where $\tilde{p}$ indicates the unconditional probability.

Note that the probabilistic description does not imply that the atom occupies two different states, but only that we do not know which one is actually occupied. This also implies that our prediction for the distribution of photon numbers $n\left(t_{i}\right)$ detected in the $i$-th time bin has to be calculated as a weighted average $\mathcal{P}(n)=\tilde{p}_{0} \mathcal{P}(n \mid 0)+(1-$ $\left.\tilde{p}_{0}\right) \mathcal{P}(n \mid 1)$. The actually measured photon counts $n\left(t_{i}\right)$ provide new information, and the state probabilities are updated using Bayes' rule of conditional probabilities:

$p_{\alpha}\left(t_{i}\right) \equiv p\left(\alpha \mid n\left(t_{i}\right)\right)=\frac{\mathcal{P}\left(n\left(t_{i}\right) \mid \alpha\right) \tilde{p}_{\alpha}\left(t_{i}\right)}{\sum_{\alpha} \tilde{p}_{\alpha}\left(t_{i}\right) \mathcal{P}\left(n\left(t_{i}\right) \mid \alpha\right)}$ for $\alpha=0$ and 1 .

The conditional probabilities $\mathcal{P}\left(n\left(t_{i}\right) \mid \alpha\right)$ are extracted from the separately measured photon count histograms for $\alpha=0$ and 1, respectively. Setting the initial probabilities to $p_{0}(0)=0, p_{1}(0)=1$, because the atom is prepared in $|F=4\rangle$ before being transported into the cavity, $p_{\alpha}\left(t_{i}\right)$ is then updated stepwise for each time bin. In this way, the time-dependent atomic state probabilities are computed successively for the whole transmission trace.

Figure 5 (a) shows an example trace of a telegraph signal to which the Bayesian algorithm was applied. Most of the time, the probability $p_{0}(t)$, plotted in (b), is close to either 0 or 1 , while narrow spikes indicate short periods of time with less complete knowledge about $p_{\alpha}$. The Bayes analysis provides more definite probabilities than a matching of the currently transmitted signal to the state dependent transmission rate, because it updates previously estimated results and thus accumulates statistical significance over time. The optical probing of the system does not, however, prevent atomic transitions from taking place, and during such transitions, the Bayes algorithm faithfully reproduces our inability to determine the state of the atom with certainty until a significant amount of data has been accumulated which is in agreement with the new state of the atom. In this context, the narrow spikes in the figure illustrate the "willingness" of the Bayesian update to interpret a few unexpected photon counts as the emerging signal of a change of state, while they may be only statistical fluctuations. For photon count histograms with negligible overlap, the Bayesian algorithm would yield the same result as a simple threshold analysis. Its main advantage is that one can still extract information about the spin dynamics even for a signal where the signal-to-noise ratio prohibits a threshold analysis, as will become apparent in the next section. 


\section{SPIN DYNAMICS OF TWO ATOMS}

So far we have presented experiments revealing the internal spin dynamics of one atom coupled to the cavity mode. Placing two atoms into the resonator leads to an effective interaction between them, mediated by the cavity field [13], and detecting the number of atoms being in a particular state could be used for entanglement generation in cavity-QED-systems [14].

\section{A. Counting the number of atoms in $|F=4\rangle$}

In the previous section the atomic state was determined from the probe laser transmission. Without changing the experimental settings, this is not directly possible for two atoms coupled to the resonator. Both atoms in $|F=3\rangle$, i.e. $\alpha=0$, will lead to a transmission level $T_{0}=1$ equal to the empty-cavity case. One atom in $|F=4\rangle$ and one in $|F=3\rangle(\alpha=1)$ will cause the transmission $T_{1}$ to drop almost to zero, which implies that $\alpha=2$ is indistinguishable from $\alpha=1$. To deduce $\alpha=0,1,2$ from the corresponding transmission levels $T_{0}, T_{1}, T_{2}$, the experimental settings have to be adapted.

In the weak excitation limit, two atoms at rest coupled with the same strength $g$ to the cavity can be theoretically described as a single atom experiencing a coupling strength $g_{2}=\sqrt{2} g$. In the dispersive limit $\left(\Delta_{c a} \gg \gamma\right)$, Eq. (11) thus yields

$$
T_{1}=\frac{1}{1+\left(\frac{g^{2}}{\kappa \Delta_{c a}}\right)^{2}}, T_{2}=\frac{1}{1+\left(\frac{2 g^{2}}{\kappa \Delta_{c a}}\right)^{2}},
$$

for the transmission levels. The level difference $\Delta T_{12}=T_{1}-T_{2}$ reaches its maximum value of $33 \%$ for $g^{2} /\left(\kappa \Delta_{c a}\right)=1 / \sqrt{2}$, where $T_{0}, T_{1}$, and $T_{2}$ are equally spaced. In order to examine this theoretical prediction experimentally, the transmission level $T_{2}$ was measured alongside the one-atom transmission. Figure 7 shows that for two atoms the transmission is lower, but instead of the theoretically expected value of $\sqrt{2} \times 9 \mathrm{MHz}$ $\approx 13 \mathrm{MHz}$, it is compatible with an effective coupling of $g_{2, \mathrm{eff}} \approx 2 \pi \times 11 \mathrm{MHz}$. As a consequence, the measured level difference $\Delta T_{12}$ is at maximum about $20 \%$ for a detuning of $\Delta_{c a}=2 \pi \times 270 \mathrm{MHz}$.

A detuning of $\Delta_{c a}$ in the range of 200 to $300 \mathrm{MHz}$ has, however, two disadvantages for studying the spindynamics of two coupled atoms: Firstly, the difference in cavity transmission is quite small compared to the noise, and secondly, the average dwell time $R_{43}^{-1}$ is close to its minimum value for $\Delta_{c a}>2 \pi \times 150 \mathrm{MHz}$, with a very shallow slope towards higher detunings, see fig. 4 (b). Closer to resonance, this time is longer, but if two atoms are at the cavity center, the transmission levels $T_{1}$ and $T_{2}$ are almost indistinguishable.

The level difference $\Delta T_{12}$ can, however, be controlled for a constant detuning $\Delta_{c a}$ by changing $g_{\text {eff. This is pos- }}$ sible by means of our optical conveyor belt, which allows

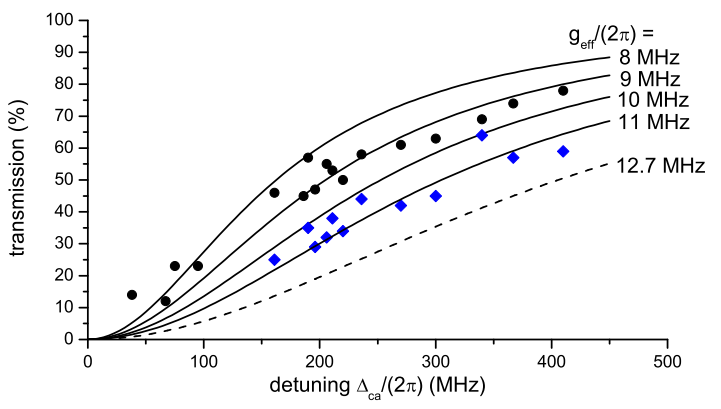

FIG. 7: Normalized transmission $T_{1}$ (black dots) and $T_{2}$ (blue diamonds) for one and two atoms, respectively. The solid lines are calculated according to the effective two-level model (11) for one atom at rest with different values for $g_{\mathrm{eff}}$, and the dashed line shows the theoretically expected two-atom transmission for $g_{\text {eff }} /(2 \pi)=\sqrt{2} \times 9=12.7 \mathrm{MHz}$. The oneatom data is the same as in fig. 4(a).

us to transport atoms not only into the cavity center, but also to stop the transport at a predetermined distance $\Delta y$ away from it. With $g_{\text {eff }}(\Delta y=0)=2 \pi \times 9 \mathrm{MHz}$, the coupling strength as a function of position along the conveyor belt axis reads $g_{\text {eff }}(\Delta y)=g_{\text {eff }}(0) \exp \left(-\Delta y^{2} / w_{0}^{2}\right)$. From Eq. (11) the required distance $\Delta y$ to achieve $\Delta T_{12}=0.33$ is calculated to be

$$
\left|\Delta y\left(\Delta_{c a}\right)\right|=w_{0} \sqrt{\frac{1}{2} \ln \left(\frac{\sqrt{2} g_{\text {eff }}^{2}(0)}{\Delta_{c a} \kappa}\right)} .
$$

For $\Delta_{c a}>2 \pi \times 280 \mathrm{MHz}, \Delta T_{12}$ is always at maximum for $\Delta y=0$, i.e. at the cavity center. Figure 8 shows the calculated level difference $\Delta T_{12}$ and the quantum jump rate $R_{43}$ as a function of detuning $\Delta_{c a}$ and distance from the cavity center $\Delta y$. By choosing a lower detuning, the scattering rate $R_{43}$ is reduced, and it is still possible to obtain optimal distinction $\Delta T_{12}$ by positioning the atoms away from the cavity center. Empirically we found that a detuning of $\Delta_{c a}=2 \pi \times 38 \mathrm{MHz}$ is a lower limit in terms of stable transmission traces. The distance of $\Delta y=21 \mu \mathrm{m}$, at which $g_{\text {eff }} /(2 \pi) \approx 3.1 \mathrm{MHz}$, was adjusted for optimum distinction of one and two atoms.

\section{B. Two-atom telegraph signal}

To study two-atom spin dynamics, two atoms loaded into the FORT were positioned at $\Delta y=21 \mu \mathrm{m}$. At this position of around one cavity-waist away from the mode center, the coupling strength depends more critical on the exact position, therefore those traces were selected for which the measured atom-atom spacing was $\leq 2 \mu \mathrm{m}$. As for the one-atom case, the repumper was attenuated to a level at which it induced quantum jumps from $|F=3\rangle$ to $|F=4\rangle$ at a rate comparable with the probe laser induced jumps. 


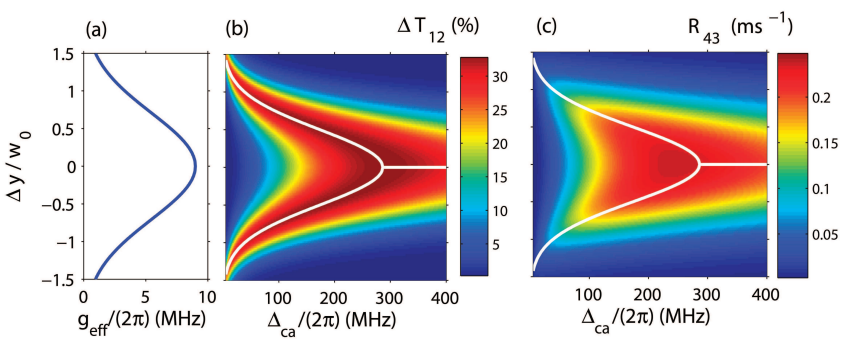

FIG. 8: (a) Effective coupling as a function of distance $\Delta y$ from the cavity center. (b) Transmission level difference $\Delta T_{12}$, (c) quantum jump rate $R_{43}$ as a function of detuning $\Delta_{c a}$ and distance $\Delta y$. The white solid lines are points of maximum $\Delta T_{12}$ calculated according to Eq. (12).

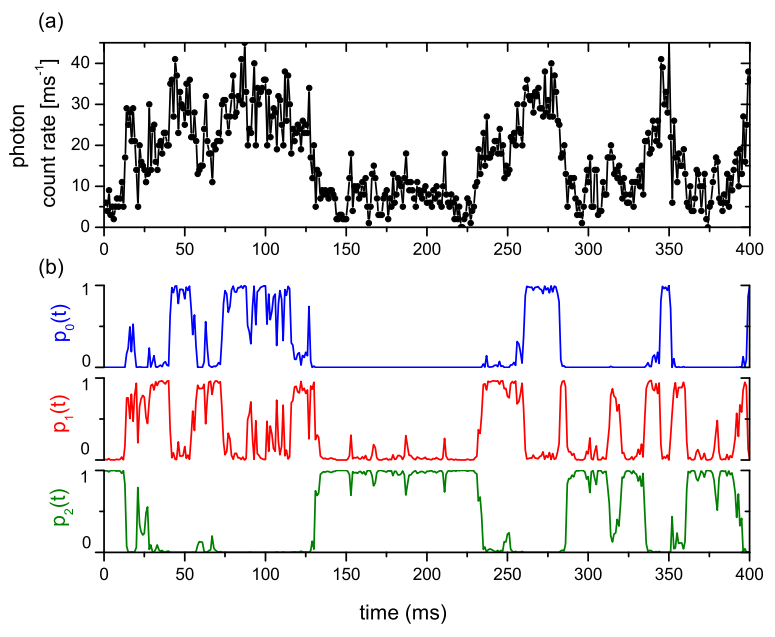

FIG. 9: (a) Example trace of a random telegraph signal for two atoms placed $\Delta y=21 \mu \mathrm{m}$ away from the cavity center. The cavity-atom detuning is $\Delta_{c a}=2 \pi \times 38 \mathrm{MHz}$. (b) Probabilities for 0,1 , or 2 atoms to be in $|F=4\rangle$, calculated using the Bayes method.

Figure 9 (a) shows an example single trace of a twoatom telegraph signal. For $t \approx 200 \ldots 300 \mathrm{~ms}$, steps corresponding to $\alpha=2$ (low transmission), $\alpha=1$ (intermediate level), and $\alpha=0$ (empty cavity transmission) are discernible, but in general the distinction between the levels is not as clear as for the one-atom case. The degree of the level separation can be deduced from a histogram extracted from several hundred telegraph signals, see fig. 10 .

This histogram does obviously not show a three-peak structure. To quantify the contributions of the transmission levels $T_{0}, T_{1}$, and $T_{2}$, we independently measured photon count histograms for 0,1 , and 2 atoms coupled to the resonator at the same position and for the same detuning as for the telegraph signals, depicted as solid lines in fig. 10. These were obtained from signals of continuously coupled atoms, i.e. a sufficiently strong repumper was applied. The photon count histogram of the telegraph signal (black line) agrees well with a fit calculated

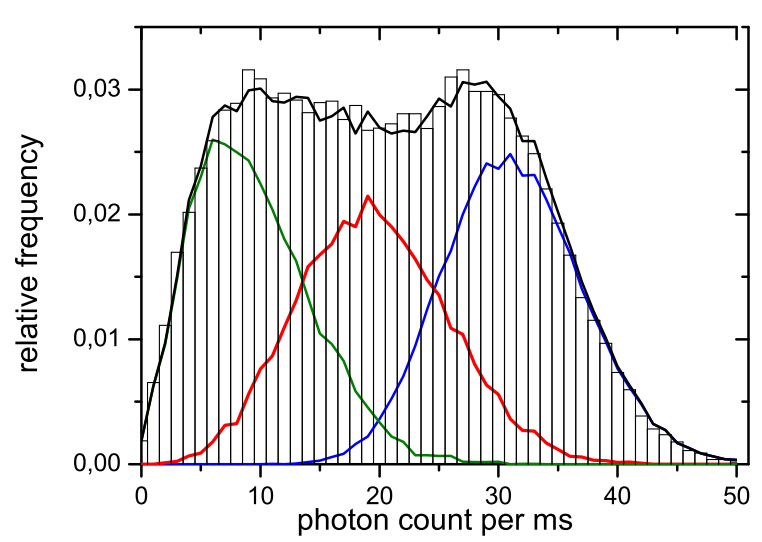

FIG. 10: Normalized photon count histogram (bars) of many two-atom telegraph signals. The blue, red, and green lines are independently measured histograms for 0,1 , and 2 atoms coupled continuously to the cavity, respectively. The black line is a weighted sum of those three histograms.

as a weighted sum of the three individual histograms conditioned on the atomic states.

The statistical analysis is performed analogous to the one atom case, but the set of rate equations now involves three atomic states and reads

$$
\begin{aligned}
& \frac{d p_{0}}{d t}=-R_{01} p_{0}(t)+R_{10} p_{1}(t) \\
& \frac{d p_{1}}{d t}=R_{01} p_{0}(t)-R_{10} p_{1}(t)-R_{12} p_{1}(t)+R_{21} p_{2}(t) \\
& \frac{d p_{2}}{d t}=R_{12} p_{1}(t)-R_{21} p_{2}(t)
\end{aligned}
$$

A transition of an atom from $|F=3\rangle$ to $|F=4\rangle$ is only induced by the repumper at a rate $R_{\text {rep }}$, which is independent of $\alpha$ because the laser is applied from the side of the cavity. Thus $R_{12}=R_{\text {rep }}$ and $R_{01}=2 R_{\text {rep }}$, because for the latter case two atoms both in $|F=3\rangle$ are present. In contrast, $R_{21}$, i.e. the rate that one out of two atoms in $|F=4\rangle$ undergoes a quantum jump to $|F=3\rangle$, is not simply given by $2 R_{10}$, because this transition is induced by the probe laser, the intensity of which depends on $\alpha$ [9]. Theoretically, the jump rate depends linearly on the intracavity intensity, thus we expect

$$
R_{21}=2 \frac{T_{2}}{T_{1}} R_{10},
$$

but this relation is not fixed for the calculation and the three rates $R_{21}, R_{21}$ and $R_{\text {rep }}$ are considered as independent parameters for the calculation. A weighted fit to the photon count histogram has two independent fit parameters and yields the steady state populations, which are related to the ratio of the three rates. In contrast to the single atom case, here it is not possible to the make a reasonable fit to the correlation function to obtain the sum of the rates and thus all three parameters. Instead, we initially guess the transition rates and employ the Bayesian 
update method to extract time dependent atomic state probabilities. Then we apply a fit as described below to iteratively extract values for the transition rates $R_{10}$, $R_{21}$ and $R_{\text {rep }}$, which ensure the optimum agreement of the time averaged probabilities with the steady state solution of the rate equations.

A good initial guess for the rate $R_{10}$ can be obtained from the transition rate for a single atom placed at the same distance $\Delta y$ away from the cavity center, with no repumper applied, similar to the measurement presented in fig. 3 (b). The transmission levels $T_{2}$ and $T_{1}$ are measured independently, which yields then an estimate for $R_{21}$ according to Eq. (16). The rate $R_{\text {rep }}$ cannot be measured independently, but since the power of the repumping laser is adjusted such that the transition rates from $|F=4\rangle$ to $|F=3\rangle$ and vice versa are approximately equal, $R_{\text {rep }}$ is set to $R_{10}$ as a starting value for the calculation.

With the initial probabilities $p_{0}(0)=0, p_{1}(0)=$ $0, p_{2}(0)=1$, the Bayesian algorithm is performed stepwise for each time bin as described for the one-atom case, yielding probabilities $p_{\alpha}(t)$. Improved values of the three transition rates are now determined by the following iterative, self-consistent method:

An analytical solution of the rate equations for $p_{0}(t)$, $p_{1}(t)$ and $p_{2}(t)$, with the initial conditions given above, yields the ensemble-averaged probabilities $\left\langle p_{\alpha}\right\rangle(t)$ with the three jump rates as parameters. Averaging over the probabilities $p_{\alpha}(t)$ obtained from the analysis of many traces provides an experimental result for $\left\langle p_{\alpha}\right\rangle(t)$, which can be fitted with the analytical solution, in which the rates $R_{10}, R_{21}$ and $R_{\text {rep }}$ are used as fit parameters. With the new values for the rates obtained in this way, the Bayes algorithm is applied over again to all experimental traces, yielding an updated set of time dependent probabilities $p_{\alpha}(t)$, which is again averaged to extract the rates, etc. The converged set of rates obtained from this analysis is

$$
R_{10}=104 \mathrm{~s}^{-1}, R_{21}=52 \mathrm{~s}^{-1}, R_{\mathrm{rep}}=45 \mathrm{~s}^{-1},
$$

and the final results for $p_{\alpha}(t)$ for the example trace are shown in fig. Fig. 9 (b). The ratio between $R_{10}$ and $R_{21}$ obtained from this iterative process does not confirm the assumption of Eq. (16), because with $T_{1} \approx 2 T_{2}$, we would expect $R_{10} \approx R_{21}$. The reason for this discrepancy remains unclear at this stage.

\section{DISCUSSION OF STATISTICAL ANALYSIS}

In this section we will address some questions arising in connection with the statistical analysis presented in the manuscript. First, we will discuss the dependence of the Bayesian atomic state analysis on the measurement data binning time, which presents interesting questions both in the case of Posissonian and non-Poissonian counting statistics. Secondly, we will discuss the possible origin of the non-Poissonian character of the photon count records

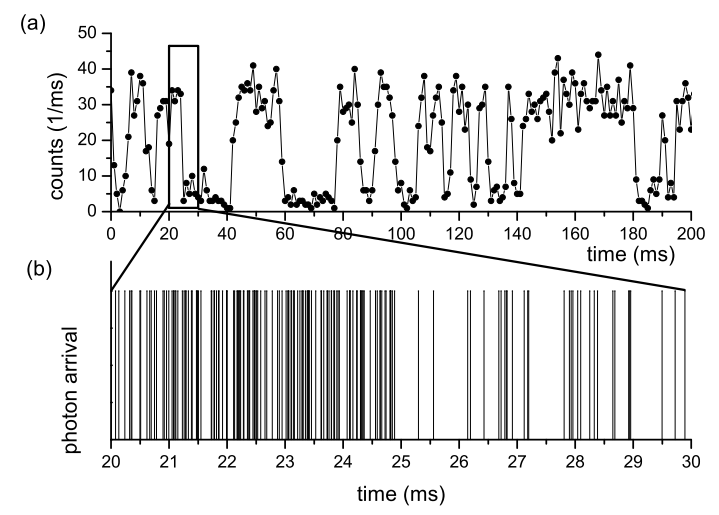

FIG. 11: (a) Random telegraph signal with 1 ms binning time. (b) Enlarged section of $10 \mathrm{~ms}$ showing photon click times. The quantum jump occurs at about $24.8 \mathrm{~ms}$.

and its consequences for our extraction of rate parameters and the Bayesian analysis.

\section{A. Bin size and optimum information extraction}

In the analysis of the one and two atom telegraph signals discussed so far, we used binning times of $1 \mathrm{~ms}$. Let us recall that the raw-data of the cavity transmission consists of a list of time intervals between photon clicks, see Fig. 11 (b) for an example trace. To study some of the consequences which a change in time bin size might have, we analyzed one and the same set of data using the Bayes formalism, but for different bin sizes.

If long time bins are used, the signal to noise ratio in each bin is good, and the count histograms for each atomic state become well separated. This implies that for long sequences of time, the atomic state probabilities will be firmly fixed to values close to zero and unity, while the instances where transitions between the states occur are not resolved within the duration of a single time bin. But this is only true as long as $\Delta t_{b}<R^{-1}$, with $R=\max \left(R_{01}, R_{10}\right)$, because for even longer times transitions will occur within a significant fraction of the bins causing considerable uncertainty about the actual atomic state.

Going to shorter time bins, the signal-to-noise ratio is decreased, and the overlap of the photon count histograms become larger. Correspondingly, it happens more frequently that a less probable, but still possible, number of counts in a time bin causes a narrow spike in the atomic state probabilities derived from the Bayes conditional update rule, where indeed no transition took place. This behavior is evident from the spikes in Fig 12 .

One would suspect that the additional information provided by subdividing data into counts registered in the first and second half of every time bin would only 
(a)

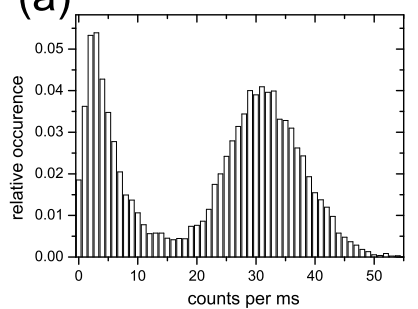

(b)

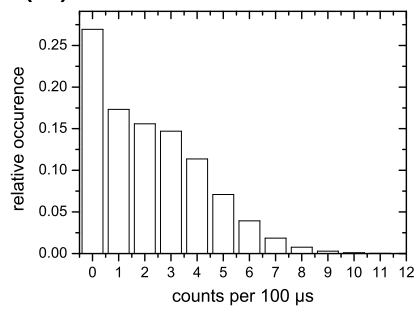

(c)

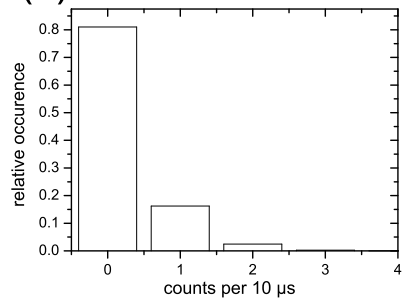

(d)

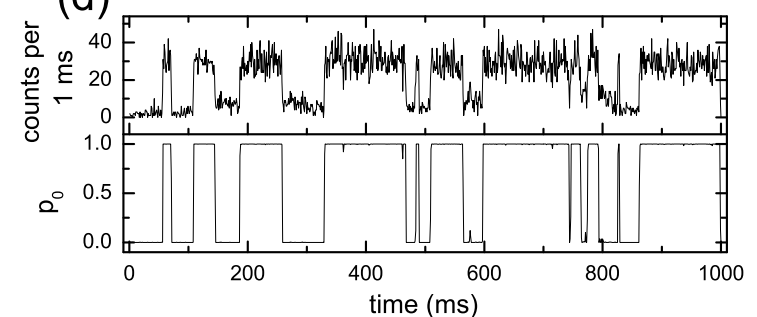

(e)

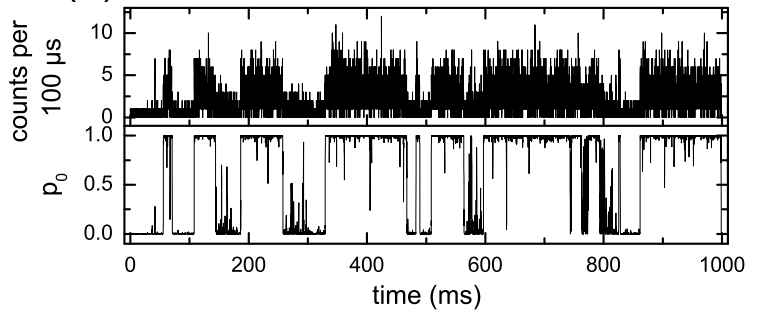

(f)

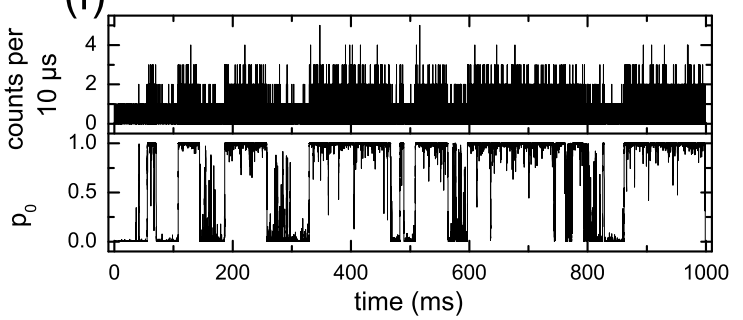

FIG. 12: Application of the Bayes algorithm for different bin times. Figures (a) to (c) show the histogram of the telegraph signal for a bin time of $1 \mathrm{~ms}, 100 \mu \mathrm{s}$, and $10 \mu \mathrm{s}$, respectively. The transmission signals, generated from the same photon-click record, and $p_{0}(t)$ are depicted in $(\mathrm{d})$ to $(\mathrm{e})$.

serve to yield a better estimate of the atomic state, since no knowledge is lost by this finer binning of the data. In the case where no transitions occur and we aim to detect the state initially occupied by the atom in a quantum nondemolition manner, the Bayesian analysis indeed becomes independent of data binning size for a Poissonian count process. To study this issue in our system, with the state-changing rate process occurring simultaneously with the probing, we evaluated the one-atom telegraph signals for different bin sizes using the Bayesian algorithm, see fig. 12. Even for $\Delta t_{b}=10 \mu \mathrm{s}$, when there is no click in $80 \%$ of all bins, the calculated probability $p_{0}(t)$ is often close to 0 or 1 , although the state probability shows more short spikes compared to $\Delta t_{b}=1 \mathrm{~ms}$.

To give a single quantitative measure of our uncertainty about the atomic state, we calculate the entropy

$$
S=\left\langle-\sum_{\alpha} p_{\alpha} \log p_{\alpha}\right\rangle
$$

where the average $\langle\ldots\rangle$ is performed over the whole duration of all analyzed traces. The entropy is plotted in fig. 13 for a range of bin times from $10 \mu \mathrm{s}$ to $20 \mathrm{~ms}$. The sharp rise of $S$ for large bins is due to the high probability in every time bin for an atomic transition to occur. We ascribe the increase of $S$ towards shorter bins to the occurrence of more spikes in $p_{\alpha}(t)$, already visible in fig. $12(\mathrm{~d})$ and (e). According to the entropy measure, there seems to be an optimum time bin, which is related to the magnitude of the quantum jump rates. We recall, however, that the entropy (18) is only one of many possible measures of the information extracted from the system. If, for example, the measurements constitute a component in a feedback mechanism, access to data on the shortest time scale may yield the better performance with respect to the desired goal of the feedback protocol.

\section{B. Origin and modeling of super-Poissonian count distributions}

The existence of an optimum bin time, leading to a minimum in the time averaged entropy (18), is observed both for our experimental histogram data and in simulations with Poissonian counting statistics associated with each atomic state. The case of super-Poissonian counting distributions, i.e., distributions with a variance exceeding the mean value of the number of counts, however, presents it own separate problems, and points to more elaborate future methods of analysis. 


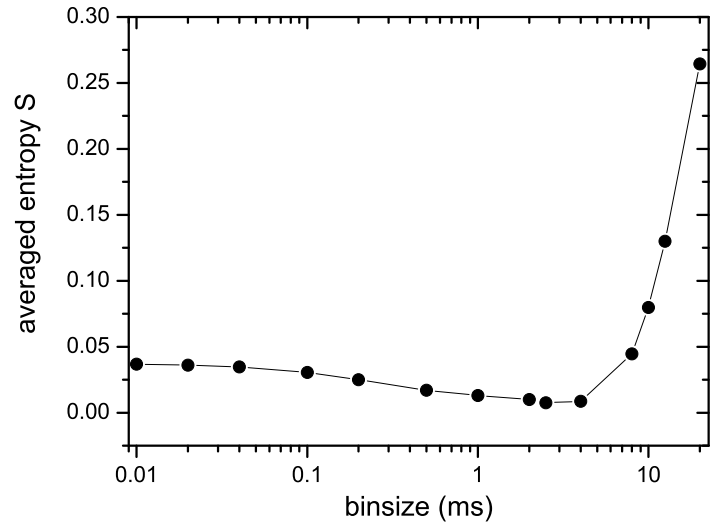

FIG. 13: Time- and ensemble averaged entropy $S$ as a function of binning time $\Delta t_{b}$.

We already commented on the apparent extra fluctuations in the light transmission signal being possibly correlated with the atomic motion between sites exhibiting different coupling strengths to the cavity mode, corresponding to different transmission levels. This suggests an extended model, where the state with no atoms coupled to the field $(\alpha=0)$ is retained as a single state, while states with $\alpha=1,2$ are split according to an extra position label, attaining a number of different values. If for example a single atom can reside in two locations leading to two different Poissonian transmission signals, the long time averaged photon count distribution will be a weighted sum of these distributions, while the count number correlation function within an experimental trace may reveal the transition rates between the atomic locations, equivalent to our analysis of internal state transitions in sec. IIIB. This is an appealing and very likely explanation of the broadened histograms, and it points to an interesting problem for our previous analysis.

If the super-Poissonian fluctuations in our counting histograms are caused by atomic motion between states with different Poissonian signals, counts in close lying time bins, where atoms have not yet moved, should be correlated. This implies that the Bayesian update is no longer a Markov process, where the updated probabilities depend only on the most recent value and the latest measurement result, but also knowledge of previous counts should be applied to extract maximum information about the atomic state. This effect may have significant consequences for very short time bins, where each bin offers a low signal-to-noise ratio, but where correlations between bins may be strong. We have analyzed our experimental records, and we indeed find such correlations, but because of limited statistics these findings could not be incorporated quantitatively into our analysis. This does not imply that our previous use of the Bayes update formalism produces erroneous results, but it should be noted that it represents the update based on a restricted access to (or memory of) the measurement data, and hence it provides a non-optimal estimate of the atomic state based on incomplete information.

\section{OUTLOOK}

We have shown that a Bayesian analysis of experimental transmission signals from a cavity containing one or two atoms provides a high degree of certainty about the atomic state. We have demonstrated how rates of the atomic processes can be fitted to the data, and we have discussed possible physical explanations of noise in the data beyond the predictions of simple models.

A natural next step would be to use a more complete model, including the larger number of position states and internal states of the atoms. We recall that already for the simplest model with only internal state dynamics, finding the parameters is not a trivial task, but ad hoc iterative procedures have allowed the identification of consistent sets of parameters used in our present analysis in this paper.

It will put stringent demands on the reproducibility of large data sets to make a reliable fit to more advanced models, but we wish to conclude this paper with a brief mentioning of a promising systematic theoretical data analysis that can be applied to such data in a future more elaborate treatment: the Hidden Markov Model (HMM) [35]. We indicated that there is a possible physical mechanism responsible for the fluctuations and for the temporal correlations between count signals. In this way we point at an underlying Markovian model, where the atoms perform transitions between different internal and position states, and for each of these states, the coherent light field is transmitted with a definite transmission coefficient, and counting statistics are Poissonian with no temporal correlations. This is, indeed, a physical realization of a Hidden Markov Process in statistical modelling of time series, with applications in insurance, finance, speech recognition, image analysis and many other fields, where a single series of data is mathematically modeled as the outcome of a system undergoing transitions between (hidden) states, each giving different data characteristics. In their most advanced forms, HMMs only assume the transitions between the hidden states to be Markovian, i.e., the state populations follow a transfer matrix of discrete or continuous population changes, while the signal can have any state dependent probability distribution.

Our problem belongs to a narrower class with continuous rate equations (with unknown rates), and it is plausible to assume Poissonian count statistics parameterized by a single parameter for each atomic state. This case is treated, e.g., in [36], and the problem of estimating the transition rates among a family of $N$ states and the $N$ photon transmission rates from the data is solved by an iterative variational application of the maximum likelihood principle. In a genuine HMM, the number of states $N$ is not known, and one merely attempts to fit the data 
with different candidate numbers of states. For an application to our problem, we are guided by the physics, and after a successful fit, we would request that the states identified should have the properties corresponding to a few position states for each of the internal state $\alpha=1,2$ cases. I.e., they should occur in groups with similar photon scattering rates, and certain transition rates should be very small or vanish.

In addition to an extended model for the analysis, we aim at improving the experimental conditions, such that the super-Poissonian noise is less pronounced. Since we attribute these fluctuations mainly to thermal motion of the atom, increasing the stability of the coupling strength requires a tighter confinement of the atom. This could be achieved by employing cavity-mediated cooling forces 33 , 34], Raman cooling [4] or additional trapping potentials.

The rate at which information about the atom-cavity system can be acquired is ultimately limited by the photon flux arriving at the detector. The most important obstacles for further enhancement of the detection efficiency are losses from the cavity-mirror coatings and the limited quantum efficiency of the SPCM. Employing homodyne or heterodyne detection would permit the use of detectors with a quantum efficiency close to $100 \%$. The former problem could be solved by using a more open cavity configuration, where the transmission coefficient is significantly larger than the losses.

Advancements both in terms of experimental conditions and statistical analysis could finally lead to the development and implementation of quantum feedback techniques for the preparation, stabilization and error correction of non-classical quantum states [37, 38].

\section{Acknowledgements}

We acknowledge financial support by the EC (IP SCALA). S. R. acknowledges support from the "Deutsche Telekom Stiftung" and T. K. acknowledges support from the "Studienstiftung des Deutschen Volkes".
[1] S. Haroche and J.-M. Raimond, Exploring the Quantum (Oxford University Press, 2006).

[2] E. T. Jaynes and F. W. Cummings, "Comparison of quantum and semiclassical radiation theories with application to the beam maser," Proc. IEEE 51, 89-109 (1963).

[3] J. McKeever, J. R. Buck, A. D. Boozer, A. Kuzmich, H.C. Nägerl, D. Stamper-Kurn, and H. J. Kimble, "Stateinsensitive cooling and trapping of single atoms in an optical cavity," Phys. Rev. Lett. 90, 133602 (2003).

[4] A. D. Boozer, A. Boca, R. Miller, T. E. Northup, and H. J. Kimble, "Cooling to the ground state of axial motion for one atom strongly coupled to an optical cavity," Phys. Rev. Lett. 97, 083602 (2006).

[5] S. Nußmann, K. Murr, M. Hijlkema, B. Weber, A. Kuhn, and G. Rempe, "Vacuum-stimulated cooling of single atoms in three dimensions," Nat. Phys. 1, 122 (2005).

[6] S. Nußmann, M. Hijlkema, B. Weber, F. Rohde, G. Rempe, and A. Kuhn, "Submicron positioning of single atoms in a microcavity," Phys. Rev. Lett. 95, 173602 (2005).

[7] K. M. Fortier, S. Y. Kim, M. J. Gibbons, P. Ahmadi, and M. S. Chapman, "Deterministic loading of individual atoms to a high-finesse optical cavity," Phys. Rev. Lett. 98, 233601 (2007).

[8] M. Khudaverdyan, W. Alt, I. Dotsenko, T. Kampschulte, K. Lenhard, A. Rauschenbeutel, S. Reick, K. Schörner, A. Widera, and D. Meschede, "Controlled insertion and retrieval of atoms coupled to a high-finesse optical resonator," N. J. Phys. 10, 073023 (2008).

[9] M. Khudaverdyan, W. Alt, T. Kampschulte, S. Reick, A. Thobe, A. Widera, and D. Meschede, "Quantum jumps and spin dynamics of interacting atoms in a strongly coupled atom-cavity system," Phys. Rev. Lett. 103, 123006 (2009).

[10] J. M. Raimond, M. Brune, and S. Haroche, "Manipulating quantum entanglement with atoms and photons in a cavity," Rev. Mod. Phys. 73, 565 (2001).

[11] A. D. Boozer, A. Boca, R. Miller, T. E. Northup, and H. J. Kimble, "Reversible state transfer between light and a single trapped atom," Phys. Rev. Lett. 98, 193601 (2007).

[12] T. Wilk, S. C. Webster, H. P. Specht, G. Rempe, and A. Kuhn, "Polarization-controlled single photons," Phys. Rev. Lett. 98, 063601 (2007).

[13] L. You, X. X. Yi, and X. H. Su, "Quantum logic between atoms inside a high-Q optical cavity," Phys. Rev. A 67, 032308 (2003).

[14] A. S. Sørensen and K. Mølmer, "Measurement induced entanglement and quantum computation with atoms in optical cavities," Phys. Rev. Lett. 91, 097905 (2003).

[15] J. Metz and A. Beige, "Macroscopic quantum jumps and entangled-state preparation," Phys. Rev. A 76, 022331 (2007).

[16] S. Gleyzes, S. Kuhr, C. Guerlin, J. Bernu, S. Deleglise, U. B. Hoff, M. Brune, J.-M. Raimond, and S. Haroche, "Quantum jumps of light recording the birth and death of a photon in a cavity," Nature 446, 297 (2007).

[17] C. Guerlin, J. Bernu, S. Deléglise, C. Sayrin, S. Gleyzes, S. Kuhr, M. Brune, J.-M. Raimond, and S. Haroche, "Progressive field-state collapse and quantum nondemolition photon counting," Nature 448, 889 (2007).

[18] Y. Yuzhelevski, M. Yuzhelevski, and G. Jung, "Random telegraph noise analysis in time domain," Review of Scientific Instruments 74, 1681 (2000).

[19] S. Kuhr, W. Alt, D. Schrader, M. Müller, V. Gomer, and D. Meschede, "Deterministic delivery of a single atom," Science 293, 278 (2001).

[20] B. Gao, "Effects of zeeman degeneracy on the steadystate properties of an atom interacting with a nearresonant laser field: Analytic results," Phys. Rev. A 48, 2443 (1993).

[21] The $m_{F}$-distribution within the $|F=4\rangle$ manifold for a coordinate system where the quantisation axis is parallel 
to the electric field, i.e. for $\pi$-transititions, is calculated to be $34.4 \%$ in $m_{F}=0,23.9 \%$ in $m_{F}= \pm 1,7.8 \%$ in $m_{F}= \pm 2,1.1 \%$ in $m_{F}= \pm 1$, and $0.1 \%$ in $m_{F}= \pm 4$.

[22] D. Schrader, I. Dotsenko, M. Khudaverdyan, Y. Miroshnychenko, A. Rauschenbeutel, and D. Meschede, "Neutral atom quantum register," Phys. Rev. Lett. 93, 150501 (2004).

[23] V. B. Braginsky, Y. I. Vorontsov, and K. S. Thorne, "Quantum nondemolition measurements," Science 209, 547 (1980).

[24] S. Chaudhury, G. A. Smith, K. Schulz, and P. S. Jessen, "Continuous nondemolition measurement of the Cs clock transition pseudospin," Phys. Rev. Lett. 96, 043001 (2006).

[25] P. J. Windpassinger, D. Oblak, P. G. Petrov, M. Kubasik, M. Saffman, C. L. G. Alzar, J. Appel, J. H. Müller, N. Jærgaard, and E. S. Polzik, "Nondestructive probing of Rabi oscillations on the Cesium clock transition near the standard quantum limit," Phys. Rev. Lett. 100, 103601 (2008).

[26] J. C. Berquist, R. G. Hulet, W. Itano, and D. J. Wineland, "Observation of quantum jumps in a single atom," Phys. Rev. Lett. 57, 1669 (1986).

[27] W. Nagourney, J. Sandberg, and H. Dehmelt, "Shelved optical electron amplifier: Observation of quantum jumps," Phys. Rev. Lett. 56, 2797 (1986).

[28] R. J. Cook and H. J. Kimble, "Possibility of direct observation of quantum jumps," Phys. Rev. Lett. 54, 1023 (1985).

[29] T. Sauter, W. Neuhauser, R. Blatt, and P. E. Toschek, "Observation of quantum jumps," Phys. Rev. Lett. 57,
1696 (1986).

[30] W. M. Itano, J. Berquist, R. G. Hulet, and D. Wineland, "Radiative decay rates in $\mathrm{Hg}^{+}$from observations of quantum jumps in a single ion," Phys. Rev. Lett. 59, 2732 (1987).

[31] G. Hechenblaikner, M. Gangl, P. Horak, and H. Ritsch, "Cooling an atom in a weakly driven high-Q cavity," Phys. Rev. A 58, 3030 (1998).

[32] R. A. Cline, J. D. Miller, M. R. Matthews, and D. J. Heinzen, "Spin relaxation of optically trapped atoms by light scattering," Optics Letters 19, 207 (1994).

[33] P. Domokos and H. Ritsch, "Mechanical effects of light in optical resonators," J. Opt. Soc. Am. B 20, 1098 (2003).

[34] K. Murr, S. Nußmann, T. Puppe, M. Hijlkema, B. Weber, S. C. Webster, A. Kuhn, and G. Rempe, "Threedimensional cavity cooling and trapping in an optical lattice," Phys. Rev. A 73, 063415 (2006).

[35] O. Cappé, E. Moulines, and T. Ryden, Inference in Hidden Markov Models (Springer, 2000).

[36] R. Paroli, G. Redaelli, and L. Spezia, "Hidden markov models for time series of overdispersed insurances counts," in "Proceedings of the XXXI International ASTIN Colloquium," (Istituto Italiano degli Attuari, Roma, 2000), p. 461.

[37] A. R. R. Carvalho and J. J. Hope, "Stabilizing entanglement by quantum-jump-based feedback," Phys. Rev. A 76, 010301 (2007).

[38] A. R. R. Carvalho, A. J. S. Reid, and J. J. Hope, "Controlling entanglement by direct quantum feedback," Phys. Rev. A 78, 012334 (2008). 